\title{
Maternal circulating status of vitamin $D$, adiponectin and lipid ratios in gestational diabetes mellitus
}

\section{ABSTRACT}

Background. The present research was an attempt to assess maternal serum values of 25 -hydroxy vitamin $D_{3}\left[25(\mathrm{OH}) D_{3}\right]$, adiponectin and the lipid ratios and comparison of their associations with insulin resistance and insulin sensitivity in gestational diabetes mellitus (GDM).

Material and methods. It was conducted on 100 participants including 49 women with GDM and 51 normal pregnant women at 24-28 weeks of gestation. Maternal fasting serum values of glucose, $25(\mathrm{OH}) \mathrm{D}_{3}$, lipid profile, adiponectin and insulin were assessed and the lipid ratios and biomarkers of resistance and sensitivity to insulin were calculated. Statistical significance was set at $P<0.05$.

Results. Current research revealed that levels of 25(OH) $D_{3}$ were significantly lower in GDM than in normal pregnancy. Binary logistic regression analysis showed that GDM was associated negatively with $25(\mathrm{OH}) \mathrm{D}_{3}$, HDL-C and adiponectin and positively with the lipid ratios. Multiple linear regression analysis showed that only values of $25(\mathrm{OH}) \mathrm{D}_{3}$ were independently associated negatively with the insulin resistance $(P=0.012)$.

Address for correspondence:

Ali Khosrowbeygi

Department of Biochemistry and Genetics

School of Medicine, Arak University

of Medical Sciences, Arak, Iran

Phone: +988634173528

Fax: +988634173529

e-mail: khosrowbeygi@yahoo.com,

a.khosrowbeygi@arakmu.ac.ir

Clinical Diabetology 2020, 9; 5: 321-327

DOI: $10.5603 /$ DK.2020.0038

Received: 15.06 .2020

Accepted: 03.08.2020
Conclusions. Levels of $25(\mathrm{OH}) \mathrm{D}_{3}$ were significantly decreased in GDM compared with normal pregnancy. On the other hand, $25(\mathrm{OH}) \mathrm{D}_{3}$ had a significant negative correlation with insulin resistance which was stronger than the lipid ratios and adiponectin. (Clin Diabet 2020; 9; 5: 321-327)

Key words: vitamin D, gestational diabetes, adiponectin, insulin resistance

\section{Introduction}

Gestational diabetes mellitus (GDM) is a kind of diabetes that is characterized with hyperglycemia during pregnancy with a prevalence of approximately 15-20 percent of all pregnancies in the world. After pregnancy, the prevalence of type 2 diabetes mellitus and cardiovascular diseases are high in women with GDM and also in their offspring [1, 2].

Vitamin D deficiency might lead to some important problems in pregnancy. Vitamin D has some regulatory functions on many of cells in addition to its traditionally been known role in calcium metabolism and bone turnover [3, 4]. Indirect relationship between vitamin $D$ insufficiency and increased risk of clinically complications such as diabetes, metabolic syndrome, obesity, hypertension and cardiovascular diseases have been reported $[2,3,5]$.

The findings of association of serum levels of vitamin D with insulin resistance and insulin sensitivity parameters in GDM are conflicting [6-9]. On the other hand, few reports are available about the relationship between levels of vitamin $D$ and the lipid ratios and comparison of the vitamin with the lipid ratios and adiponectin as predictors of insulin resistance and insulin sensitivity. 
Table 1. Comparison of demographic characteristics and serum values of biochemichal variables between women with GDM and normal pregnant women

\begin{tabular}{lccc}
\hline Parameter & Normal pregnant $(\mathbf{n}=\mathbf{5 1})$ & GDM $(\mathbf{n}=\mathbf{4 9})$ & P \\
\hline Age (years) & $29.22 \pm 0.64$ & $31.61 \pm 0.56$ & 0.006 \\
Gestational age (weeks) & $25.96 \pm 0.21$ & $26.49 \pm 0.21$ & 0.076 \\
BMI $\left[\mathrm{kg} / \mathrm{m}^{2}\right]$ & $25.21 \pm 0.34$ & $25.38 \pm 0.31$ & 0.486 \\
FBG $[\mathrm{mg} / \mathrm{dL}]$ & $78.49 \pm 1.13$ & $103.18 \pm 2.27$ & $<0.001$ \\
TG $[\mathrm{mg} / \mathrm{dL}]$ & $214.45 \pm 12.83$ & $248.11 \pm 16.51$ & 0.163 \\
TC $[\mathrm{mg} / \mathrm{dL}]$ & $239.33 \pm 8.45$ & $220.05 \pm 8.75$ & 0.066 \\
HDL-C $[\mathrm{mg} / \mathrm{dL}]$ & $53.06 \pm 2.25$ & $32.15 \pm 2.50$ & $<0.001$ \\
LDL-C $[\mathrm{mg} / \mathrm{dL}]$ & $143.38 \pm 6.76$ & $138.28 \pm 8.61$ & 0.398 \\
TG/HDL-C ratio & $4.86 \pm 0.73$ & $10.24 \pm 1.21$ & $<0.001$ \\
TC/HDL-C ratio & $5.02 \pm 0.42$ & $8.96 \pm 0.78$ & $<0.001$ \\
LDL-C/HDL-C ratio & $3.04 \pm 0.29$ & $5.92 \pm 0.63$ & $<0.001$ \\
Insulin $[\mu l \mathrm{lU} / \mathrm{mL}]$ & $7.91 \pm 1.10$ & $13.30 \pm 1.93$ & 0.016 \\
HOMA-IR & $1.51 \pm 0.21$ & $3.41 \pm 0.50$ & $<0.001$ \\
QUICKI & $0.31 \pm 0.004$ & $0.28 \pm 0.005$ & $<0.001$ \\
Adiponectin $[\mu \mathrm{g} / \mathrm{mL}]$ & $6.45 \pm 0.71$ & $4.13 \pm 0.33$ & $<0.001$ \\
$25(\mathrm{OH}) \mathrm{D}_{3}[\mathrm{ng} / \mathrm{mL}]$ & $17.62 \pm 2.01$ & $12.98 \pm 1.10$ & 0.035 \\
\hline
\end{tabular}

Data are presented as mean \pm SEM. BMI — body mass index; FBG — fasting blood glucose; TG — triglyceride; TC — total cholesterol; HDL-C — high-density lipoprotein cholesterol; LDL-C — low-density lipoprotein cholesterol; HOMA-IR — the homeostasis model assessment of insulin resistance; QUICKI - the quantitative insulin sensitivity check index; $25(\mathrm{OH}) \mathrm{D}_{3}-25$-hydroxy vitamin $\mathrm{D}_{3}$

Therefore, the aims of the current study were to assess maternal serum values of vitamin $D$, adiponectin and the lipid ratios and comparison of their associations with insulin resistance and insulin sensitivity in GDM.

\section{Material and methods}

This case-control study was approved by the Institutional Ethical Review Board of Lorestan University of Medical Sciences (LUMS.REC.1395.223). It has been reported that Iranian pregnant women are suffering from vitamin $D$ deficiency with a prevalence of $42 \%$, $56 \%$ and $81 \%$ based on cutoff values of 10,20 and 30 $\mathrm{ng} / \mathrm{mL}$, respectively. Therefore, proper supplementation with vitamin $D$ has been advised to prevent side effects of its deficiency during pregnancy [10].

According to the $75-\mathrm{g}$ oral glucose tolerance test [11] to detect GDM, case (49 nulliparous newly diagnosed GDM before insulin or any drug treatment) and control (51 nulliparous normal pregnant women) subjects were enrolled in the current study after assignment of informed consent. Blood samples were obtained at 24-28 weeks of gestation. The current study was performed in spring season. Treatment with insulin, using vitamin $D$ supplements, preeclampsia, multiple gestation, type 1 or 2 diabetes mellitus, chronic hypertension, body mass index (BMI) $>30 \mathrm{~kg} / \mathrm{m}^{2}$ and smoking were exclusion criteria of the current study [7].

Fasting maternal serum levels of glucose (FBG) and lipid profile including triglyceride (TG), total cholesterol
(TC) and high-density lipoprotein cholesterol (HDL-C) were measured (Hitachi, Germany), while low-density lipoprotein cholesterol (LDL-C) and the lipid ratios including LDL-C/HDL-C, TG/HDL-C and TC/HDL-C were calculated $[12,13]$. 25-hydroxy vitamin $\mathrm{D}_{3}\left[25(\mathrm{OH}) \mathrm{D}_{3}\right]$ (IDS Ltd, Boldon, Tyne \& Wear, UK), adiponectin (BioVendor Laboratory Medicine, Inc. Czech Republic) and insulin (Monobind Inc., USA) were measured by ELISA method (STAT FAX 3200, USA). Insulin resistance and insulin sensitivity were estimated using the homeostasis model assessment of insulin resistance (HOMA-IR) index [14] and the quantitative insulin sensitivity check index (QUICKI) [15] formulas, respectively.

The Student t-test, Pearson's correlation analysis, multiple linear regression analysis and binary logistic regression analysis were used to do statistical analyses using SPSS 19 software (SPSS Inc, Chicago, IL, USA). The collected data were expressed as mean \pm standard error of mean (SEM) and were considered statistically significant at a $P$ value less than 0.05 .

\section{Results}

Comparison of demographic characteristics between control and case subjects are given in Table 1 that shows no differences in age and BMI between two groups while case women had higher values of age. Biochemical characteristics of case and control groups were also summarized in Table 1. Values of fasting blood glucose (FBG), the lipid ratios including 

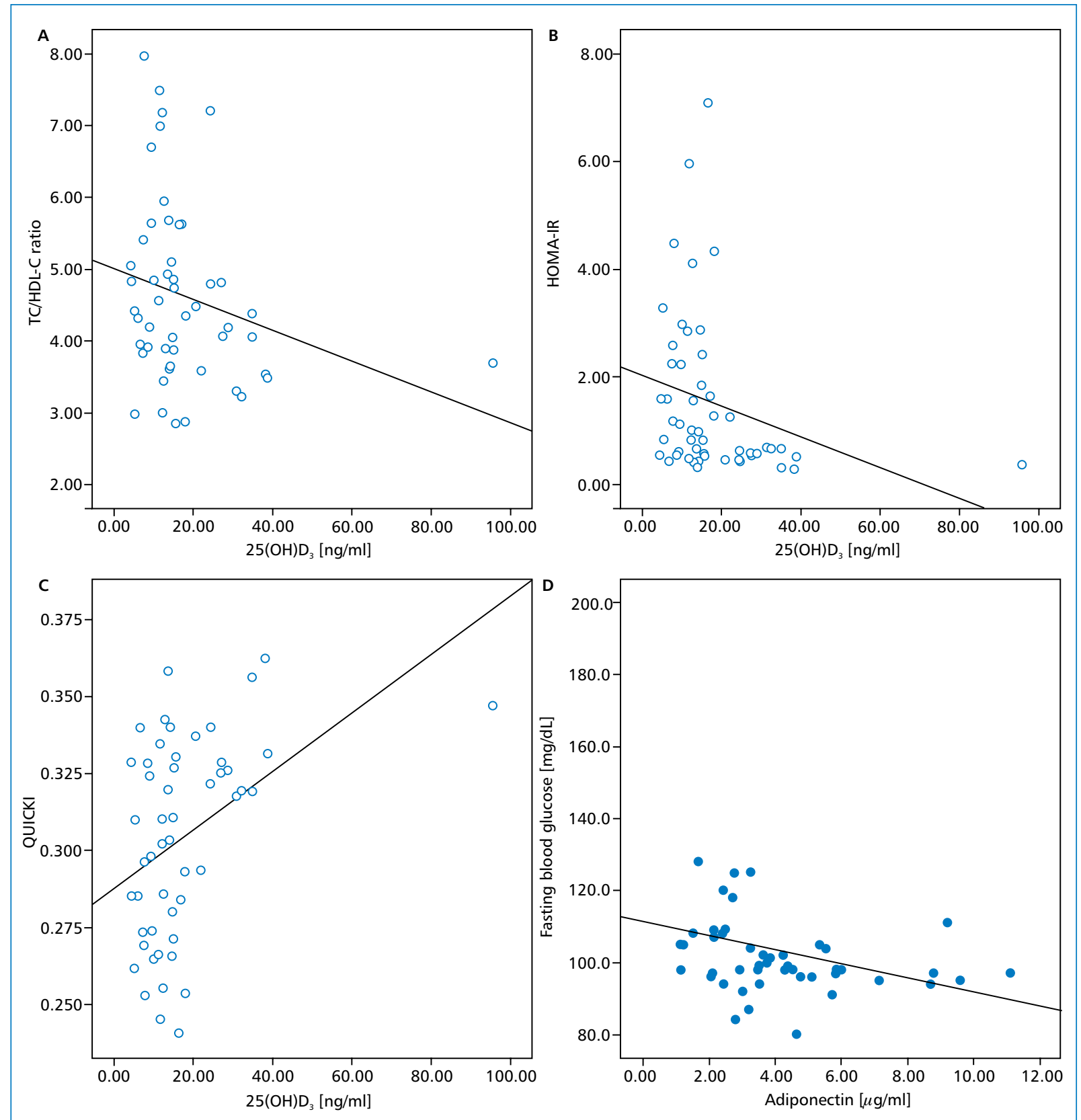

Figure 1. Correlations between serum levels of 25-hydroxy vitamin $\mathrm{D}_{3}\left(25(\mathrm{OH}) \mathrm{D}_{3}\right)$ and values of $(\mathbf{A})$ the total cholesterol/high-density lipoprotein cholesterol ratio (TC/HDL-C) $(r=-0.31, P=0.029)(B)$ the homeostasis model assessment of insulin resistance (HOMA-IR) index $(r=-0.39, P=0.005)(C)$ the quantitative insulin sensitivity check index (QUICKI) $(r=0.421, P=0.002)$ in 51 normal pregnant women and (D) correlation between fasting blood glucose and adiponectin $(r=-0.39, P=0.005)$ in 49 women with gestational diabetes mellitus

LDL-C/HDL-C, TG/HDL-C and TC/HDL-C, and the HOMA-IR index were significantly increased while values of HDL-C, QUICKI, adiponectin and $25(\mathrm{OH}) \mathrm{D}_{3}$ were significantly decreased in the case women compared with normal control group. The differences of $25(\mathrm{OH})$ $\mathrm{D}_{3}$ and adiponectin between the two groups were still statistically significant after the raw data were adjusted for the subjects' age.
In normal pregnant women, serum levels of $25(\mathrm{OH})$ $D_{3}$ correlated negatively with TC/HDL-C $(r=-0.31$, $P=0.029)$ (Fig. 1A) and HOMA-IR index $(r=-0.39$, $P=0.005)$ (Fig. 1B) and positively with QUICKI $(r=0.421$, $P=0.002$ ) (Fig. 1C). The HOMA-IR index correlated negatively with HDL-C $(r=-0.58, \mathrm{P}<0.001)$ and positively with TG/HDL-C ( $r=0.40, \mathrm{P}=0.004)$ and TC/HDL-C $(r=0.34, P=0.015)$. The QUICKI showed a positive 

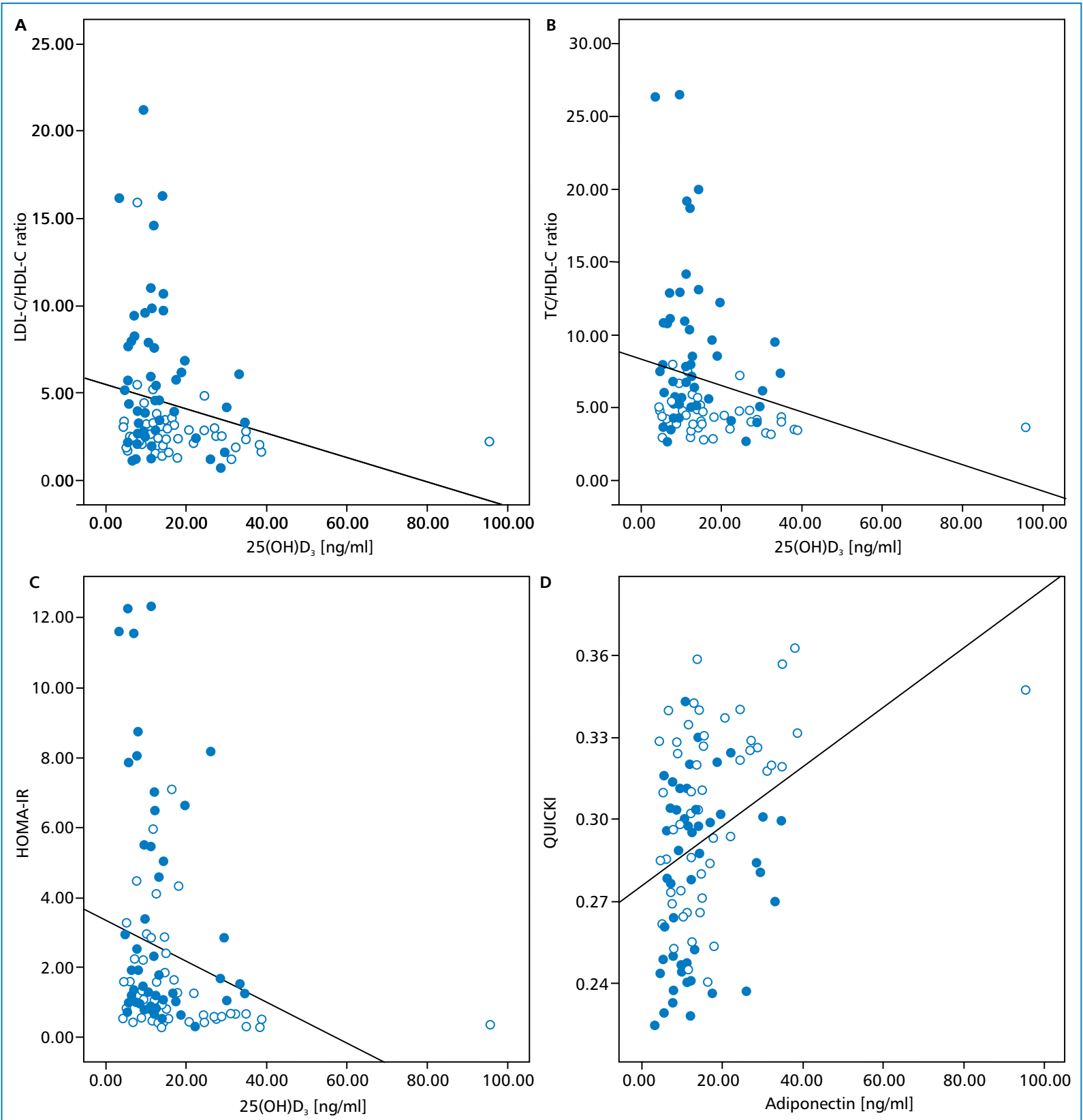

Figure 2. Correlations between serum levels of 25-hydroxy vitamin $D_{3}\left[25(\mathrm{OH}) \mathrm{D}_{3}\right]$ and values of $(A)$ the low-density lipoprotein cholesterol/high-density lipoprotein cholesterol ratio (LDL-C/HDL-C) $(r=-0.23, \mathrm{P}=0.022)(\mathrm{B})$ the total cholesterol/high-density lipoprotein cholesterol ratio $(T C / H D L-C)(r=-0.24, P=0.018)(C)$ the homeostasis model assessment of insulin resistance (HOMA$-I R)$ index $(r=-0.35, P<0.001)$ (D) the quantitative insulin sensitivity check index (QUICKI) $(r=0.37, P<0.001)$ in the whole study population including $(O) 51$ normal pregnant and $(\bullet) 49$ gestational diabetes mellitus women

correlation with HDL-C $(r=0.58, \mathrm{P}<0.001)$ and negative correlations with TG/HDL-C $(r=-0.37, \mathrm{P}=0.007)$ and TC/HDL-C ( $r=-0.31, P=0.025)$. In GDM, fasting blood glucose correlated negatively with adiponectin ( $r=-0.39, P=0.005)$ (Fig. 1D) and positively with TG/ /HDL-C ( $r=0.36, \mathrm{P}=0.011)$.

Whole study population $(n=100)$ correlations were also analyzed. Serum levels of $25(\mathrm{OH}) \mathrm{D}_{3}$ showed negative correlations with LDL-C/HDL-C $(r=-0.23$,
$P=0.022)$ (Fig. 2A), TC/HDL-C $(r=-0.24, P=0.018)$ (Fig. 2B) and HOMA-IR ( $r=-0.35, \mathrm{P}<0.001)$ (Fig. 2C) and positive correlation with QUICKI $(r=0.37, P<0.001)$ (Fig. 2D). The HOMA-IR index correlated negatively with HDL-C $(r=-0.43, P<0.001)$ and positively with LDL-C/HDL-C $(r=0.23, P=0.022), \mathrm{TG} / \mathrm{HDL}-\mathrm{C}(r=0.41$, $P<0.001)$, TC/HDL-C $(r=0.32, P=0.001)$. The QUICKI showed a positive correlation with HDL-C $(r=0.44$, $\mathrm{P}<0.001)$ and negative correlations with TG/HDL-C 
Table 2. Binary logistic regression analysis of the effects of the variables on gestational diabetes mellitus

\begin{tabular}{lcccc}
\hline Variables & B & OR & $95 \%$ Cl for OR & P \\
\hline TG/HDL-C ratio & 0.22 & 1.24 & $1.10-1.41$ & 0.001 \\
TC/HDL-C ratio & 0.33 & 1.39 & $1.15-1.68$ & 0.001 \\
LDL-C/HDL-C ratio & 0.36 & 1.43 & $1.16-1.78$ & 0.001 \\
HDL-C & -0.074 & 0.93 & $0.90-0.96$ & $<0.001$ \\
Adiponectin & -0.28 & 0.76 & $0.62-0.92$ & 0.005 \\
25(OH)D & -0.78 & 0.46 & $0.21-0.99$ & 0.046 \\
\hline
\end{tabular}

$\mathrm{Cl}$ - confidence interval; $\mathrm{OR}$ - odds ratio; other abbreviations are given in Table 1

$(r=-0.34, P=0.001)$ and TC/HDL-C $(r=-0.25$, $P=0.012)$. Fasting blood glucose showed negative correlation with adiponectin $(r=-0.32, \mathrm{P}=0.001)$ and positive correlations with TG/HDL-C $(r=0.56$, $\mathrm{P}<0.001)$, LDL-C/HDL-C $(r=0.33, \mathrm{P}=0.001)$ and $\mathrm{TC} /$ /HDL-C $(r=0.43, \mathrm{P}<0.001)$.

Multiple linear regression analysis showed that only values of $25(\mathrm{OH}) \mathrm{D}_{3}$ were independently associated with the HOMA-IR index $(\beta=-0.25, \mathrm{P}=0.012)$ and the QUICKI $(\beta=0.27, \mathrm{P}=0.005)$.

According to binary logistic regression analysis, the lipid ratios were positive risk factors for GDM development while $\mathrm{HDL}-\mathrm{C}$, adiponectin and $25(\mathrm{OH}) \mathrm{D}_{3}$ were negative risk factors (Table 2 ).

\section{Discussion}

In the current study we observed that values of $25(\mathrm{OH}) \mathrm{D}_{3}$ decreased significantly in GDM compared with normal pregnancy and $25(\mathrm{OH}) \mathrm{D}_{3}$ significantly associated with insulin resistance, insulin sensitivity and atherogenic indexes.

Decreased maternal circulating levels of $25(\mathrm{OH}) \mathrm{D}_{3}$ in GDM compared with normal pregnancy in the current study were in line with previous studies including Maghbooli et al. [6], Ou et al. [7] and McManus et al. [16]. On the other hand, the current study was contradicted to studies of El Lithy et al. [8], Mutlu et al. [9] and Pleskačová et al. [17], who have reported no significant differences in maternal circulating levels of $25(\mathrm{OH}) \mathrm{D}_{3}$ between GDM and normal pregnancy. A major limitation of some of these studies was that BMI was not similar between case and control group [6, 7, 17], since it has been reported a negative correlation between serum levels of $25(\mathrm{OH}) \mathrm{D}_{3}$ and BMI. Therefore, it has been concluded that this negative correlation is due to this fact that most of vitamin $D$ is stored in fat tissue [18].

Study of Takhshid et al. [14] showed that the HOMA-IR index is increased in GDM compared with normal pregnancy while no significant differences were observed in serum values of lipid profile, TG/ /HDL-C, adiponectin and the QUICKI between the two groups. On the other hand, current study confirmed decreased levels of both $25(\mathrm{OH}) \mathrm{D}_{3}$ and adiponectin in GDM that has been reported by McManus et al. [16]. The low levels of both $25(\mathrm{OH}) \mathrm{D}_{3}$ and adiponectin that was observed in the current study could be interpreted that vitamin $D$ has inducing effects on adiponectin gene expression [18]. Anti-inflammatory, insulin sensitivity enhancing and atherosclerosis protecting effects of adiponectin have been reported previously [19]. Some antioxidant properties have been shown for vitamin D [20] and adiponectin [21, 22]. On the other hand, it has been reported that vitamin $D$ has some beneficial effects against cardiovascular diseases which could be due to its positive effects on adiponectin's gene expression [18]. Therefore, decreased levels of adiponectin in women with GDM that was observed in the current study could be addressed that they had vitamin D deficiency.

No significant differences in serum values of lipid profile and the HOMA-IR index have been observed between GDM and normal pregnancy in a study that was conducted by Limura et al. [23]. They also observed that plasma lipid profile cannot be used to predict GDM. On the other hand, study of Wang et al. [24] showed that values of the TG/HDL-C ratio are higher in GDM than in normal pregnancy and the ratio is associated with the risks of GDM. Therefore, they concluded that for prediction of GDM risk the TG/HDL-C ratio can be used as a marker. The findings of the current study were in line with Wang et al. findings. Another study showed that higher values of TG in early pregnancy are associated with higher risk for developing GDM while higher values of HDL are associated with lower risk for the disease [25]. Study of Liang et al. [26] showed that circulating levels of TG and TC are increased in GDM compared with normal pregnancy and they are correlated directly with the HOMA-IR index. Liang et al. [26] concluded that maternal complications result from GDM could be improved partially by reducing insulin resistance and circulating lipids. In the current study, the HOMA-IR index showed a negative correlation with HDL-C and positive correlations with all the lipid ratios and insulin sensitivity was lower in GDM than in normal pregnancy that confirmed Liang et al. [26] study. In the current study, multiple linear regression analysis of the dependent variables including HOMA-IR and QUICKI with values of $25(\mathrm{OH}) \mathrm{D}_{3}$, adiponectin and the lipid ratios was also performed. The regression model showed that only values of $25(\mathrm{OH}) \mathrm{D}_{3}$ were independently associated with both HOMA-IR and QUICKI. Previous 
studies have been reported that maternal serum levels of $25(\mathrm{OH}) \mathrm{D}_{3}$ are correlated inversely with the HOMA-IR index $[6,7,9]$ that was confirmed by the current study. We also observed a positive correlation between levels of $25(\mathrm{OH}) \mathrm{D}_{3}$ and QUICKI that did not agree with Maghbooli et al. [6] and El Lithy et al. [8] studies, who have not observed any significant correlation.

It has been reported that transport of glucose is increased into insulin-dependent cells by stimulating expression of insulin receptors in the presence of vita$\min D$ that leads to increased insulin sensitivity $[2,27]$.

Formation of foam cells and uptake of cholesterol by macrophages have been reported that are inhibited in the presence of vitamin D. This property of vitamin D is considered as its antiatherogenic property [5]. On the other hand, indirect correlalation has been reported between serum level of $25(\mathrm{OH}) \mathrm{D}_{3}$ and metabolic syndrome biomarkers [28]. There are little findings about the association between the lipid ratios and $25(\mathrm{OH}) \mathrm{D}_{3}$ in GDM. In the current study, maternal serum levels of $25(\mathrm{OH}) \mathrm{D}_{3}$ showed inverse correlations with the lipid ratios that confirmed the antiatherogenic properties of vitamin D.

\section{Conclusions}

In summary, serum levels of $25(\mathrm{OH}) \mathrm{D}_{3}$ were decreased significantly in GDM compared with normal pregnancy and showed statistically significant correlations with metabolic syndrome biomarkers. $25(\mathrm{OH}) \mathrm{D}_{3}$ had more significant correlations with insulin resistance and sensitivity indexes than the lipid ratios and adiponectin.

\section{Acknowledgments}

This work was supported by Lorestan University of Medical Sciences. The authors should appreciate all patients who participated in the current study.

\section{Conflict of interest}

The authors declared that they have no conflict of interest.

\section{REFERENCES}

1. Korkmazer $E$, Solak N. Correlation between inflammatory markers and insulin resistance in pregnancy. J Obstet Gynaecol. 2015; 35(2): 142-145, doi: 10.3109/01443615.2014.948408, indexed in Pubmed: 25111421.

2. Zhang MX, Pan GT, Guo JF, et al. Vitamin D deficiency increases the risk of gestational diabetes mellitus: a meta-analysis of observational studies. Nutrients. 2015; 7(10): 8366-8375, doi: 10.3390/nu7105398, indexed in Pubmed: 26437429.

3. Rutkowska J, Bandurska-Stankiewicz E, Wiatr-Bykowska D, et al. Vitamin D deficiency in women with gestational diabetes mellitus. Clin Diabetol. 2016; 5(2): 57-61, doi: 10.5603/dk.2016.0010.
4. Rizzo G, Garzon S, Fichera M, et al. Vitamin D and gestational diabetes mellitus: is there a link? Antioxidants (Basel). 2019; 8(11), doi: 10.3390/antiox8110511, indexed in Pubmed: 31731439.

5. Mozos I, Marginean O. Links between Vitamin D deficiency and cardiovascular diseases. Biomed Res Int. 2015; 2015: 109275, doi: 10.1155/2015/109275, indexed in Pubmed: 26000280.

6. Maghbooli Z, Hossein-Nezhad A, Karimi F, et al. Correlation between vitamin D3 deficiency and insulin resistance in pregnancy. Diabetes Metab Res Rev. 2008; 24(1): 27-32, doi: 10.1002/ dmrr.737, indexed in Pubmed: 17607661.

7. Wang Ou, Nie M, Hu YY, et al. Association between vitamin D insufficiency and the risk for gestational diabetes mellitus in pregnant Chinese women. Biomed Environ Sci. 2012; 25(4): 399-406, doi: 10.3967/0895-3988.2012.04.004, indexed in Pubmed: 23026519.

8. El Lithy A, Abdella RM, El-Faissal YM, et al. The relationship between low maternal serum vitamin $D$ levels and glycemic control in gestational diabetes assessed by $\mathrm{HbA} 1 \mathrm{c}$ levels: an observational cross-sectional study. BMC Pregnancy Childbirth. 2014; 14: 362, doi: 10.1186/1471-2393-14-362, indexed in Pubmed: 25308347.

9. Mutlu N, Esra H, Begum A, et al. Relation of maternal vitamin D status with gestational diabetes mellitus and perinatal outcome. Afr Health Sci. 2015; 15(2): 523-531, doi: 10.4314/ahs.v15i2.27, indexed in Pubmed: 26124799.

10. Badfar G, Shohani M, Mansouri A, et al. Vitamin D status in Iranian pregnant women and newborns: a systematic review and meta-analysis study. Expert Rev Endocrinol Metab. 2017; 12(5): 379-389, doi: 10.1080/17446651.2017.1365596, indexed in Pubmed: 30058894.

11. American Diabetes Association. Diagnosis and classification of diabetes mellitus. Diabetes Care. 2014; 37 Suppl 1: S81-S90, doi: 10.2337/dc14-S081, indexed in Pubmed: 24357215.

12. Friedewald WT, Levy RI, Fredrickson DS. Estimation of the concentration of low-density lipoprotein cholesterol in plasma, without use of the preparative ultracentrifuge. Clin Chem. 1972; 18(6): 499-502, indexed in Pubmed: 4337382.

13. Zhang L, Chen S, Deng A, et al. Association between lipid ratios and insulin resistance in a Chinese population. PLoS One. 2015; 10(1): e0116110, doi: 10.1371/journal.pone.0116110, indexed in Pubmed: 25635876.

14. Takhshid MA, Haem Z, Aboualizadeh F. The association of circulating adiponectin and $+45 \mathrm{~T} / \mathrm{G}$ polymorphism of adiponectin gene with gestational diabetes mellitus in Iranian population. J Diabetes Metab Disord. 2015; 14: 30, doi: 10.1186/s40200-015-0156-z, indexed in Pubmed: 25909078.

15. Katz A, Nambi SS, Mather K, et al. Quantitative insulin sensitivity check index: a simple, accurate method for assessing insulin sensitivity in humans. J Clin Endocrinol Metab. 2000; 85(7): 2402-2410, doi: 10.1210/jcem.85.7.6661, indexed in Pubmed: 10902785.

16. McManus $R$, Summers $K$, de Vrijer $B$, et al. Maternal, umbilical arterial and umbilical venous 25 -hydroxyvitamin D and adipocytokine concentrations in pregnancies with and without gestational diabetes. Clin Endocrinol (Oxf). 2014; 80(5): 635-641, doi: 10.1111/cen.12325, indexed in Pubmed: 24102192.

17. Pleskačová $A$, Bartáková V, Pácal $L$, et al. Vitamin $D$ status in women with gestational diabetes mellitus during pregnancy and postpartum. Biomed Res Int. 2015; 2015: 260624, doi: 10.1155/2015/260624, indexed in Pubmed: 26000285.

18. Kardas F, Kendirci M, Kurtoglu S. Cardiometabolic risk factors related to vitamin $D$ and adiponectin in obese children and adolescents. Int J Endocrinol. 2013; 2013: 503270, doi: 10.1155/2013/503270, indexed in Pubmed: 23983686.

19. Frühbeck G, Catalán V, Rodríguez $A$, et al. Involvement of the leptin-adiponectin axis in inflammation and oxidative stress in the metabolic syndrome. Sci Rep. 2017; 7(1): 6619, doi: 10.1038/ s41598-017-06997-0, indexed in Pubmed: 28747790. 
20. Kostoglou-Athanassiou I, Athanassiou P, Gkountouvas A, et al. Vitamin $D$ and glycemic control in diabetes mellitus type 2. Ther Adv Endocrinol Metab. 2013; 4(4): 122-128, doi: 10.1177/2042018813501189, indexed in Pubmed: 23997931.

21. Khosrowbeygi A, Ahmadvand $H$. Leptin to adiponectin ratio in preeclampsia. Bangladesh Med Res Counc Bull. 2013; 39(1): 18-21, doi: 10.3329/bmrcb.v39i1.15805, indexed in Pubmed: 23923407.

22. Ren Y, Li Y, Yan J, et al. Adiponectin modulates oxidative stressinduced mitophagy and protects C2C12 myoblasts against apoptosis. Sci Rep. 2017; 7(1): 3209, doi: 10.1038/s41598-01703319-2, indexed in Pubmed: 28600493.

23. limura Y, Matsuura M, Yao Z, et al. Lack of predictive power of plasma lipids or lipoproteins for gestational diabetes mellitus in Japanese women. J Diabetes Investig. 2015; 6(6): 640-646, doi: 10.1111/jdi.12363, indexed in Pubmed: 26543537.

24. Wang D, Xu S, Chen $H$, et al. The associations between triglyceride to high-density lipoprotein cholesterol ratios and the risks of gestational diabetes mellitus and large-for-gestational-age infant. Clin Endocrinol (Oxf). 2015; 83(4): 490-497, doi: 10.1111/ cen.12742, indexed in Pubmed: 25665068.

25. Li G, Kong L, Zhang Li, et al. Early pregnancy maternal lipid profiles and the risk of gestational diabetes mellitus stratified for body mass index. Reprod Sci. 2015; 22(6): 712-717, doi: 10.1177/1933719114557896, indexed in Pubmed: 25394643.

26. Liang $Z x, W u Y, Z$ hu $X y$, et al. Insulin resistance and lipid profile during an oral glucose tolerance test in women with and without gestational diabetes mellitus. J Obstet Gynaecol. 2016; 36(3): 337-339, doi: 10.3109/01443615.2015.1060197, indexed in Pubmed: 26466813.

27. Hu L, Zhang $Y$, Wang $X$, et al. Maternal Vitamin D status and risk of gestational diabetes: a meta-analysis. Cell Physiol Biochem. 2018; 45(1): 291-300, doi: 10.1159/000486810, indexed in Pubmed: 29402818.

28. Barchetta I, De Bernardinis M, Capoccia D, et al. Hypovitaminosis $D$ is independently associated with metabolic syndrome in obese patients. PLoS One. 2013; 8(7): e68689, doi: 10.1371/journal. pone.0068689, indexed in Pubmed: 23935881. 